\title{
Protocolo ultrasonográfico enfocado en cirugía cardiaca posquirúrgica CCROSS (Cardiac, Cerebral, Renal, Optic nerve, lung ultraSound Study)
}

\author{
Ultrasonographic protocol focused on post cardiac surgery CCROSS (Cardiac, Cerebral, \\ Renal, Optic nerve, lung ultraSound Study)
}

Jessica Garduño-López*, Edgar García-Cruz y Francisco M. Baranda-Tovar

Unidad de Terapia Intensiva Cardiovascular, Instituto Nacional de Cardiología Ignacio Chávez Ciudad de México, México

\begin{abstract}
Resumen
El uso de ecocardiografía enfocada es de gran utilidad en la valoración, tratamiento y seguimiento del paciente en estado crítico. Es, junto con la clínica y el estetoscopio, una herramienta que complementa el actuar del médico ante las diversas etiologías que determinan un estado de choque o aumentan la morbimortalidad, especialmente en pacientes posquirúrgicos de cirugía cardíaca, en quienes no se tiene algoritmos de manejo emergente en el posquirúrgico. Ante tal situación, en el Instituto Nacional de Cardiología Ignacio Chávez se ha realizado y propuesto un algoritmo de manejo en los pacientes posquirúrgicos cardíacos: mediante ultrasonografía enfocada, abarcando ecoscopia transtorácica, ultrasonido pulmonar, ultrasonido del nervio óptico y renal mediante la valoración de índices resistivos renales. Diversas sociedades han creado sus protocolos de abordaje en pacientes en estado crítico, por lo que en el Instituto, específicamente en la terapia intensiva cardiovascular, ha creado el protocoIo CCROSS (Cardiac, Cerebral, Renal, Optic nerve, lung ultraSound Study) para el abordaje inicial de estos pacientes y se encuentra en marcha actualmente un estudio para su validación, reproducibilidad y eficacia.
\end{abstract}

Palabras clave: Ultrasonido enfocado. Cirugía cardíaca. Posquirúrgico inmediato. Unidad de cuidados críticos. México.

\begin{abstract}
The use of echocardiography is very useful in the evaluation, treatment and follow-up of the patient in critical condition. Along with clinic and the stethoscope, it is a tool that complements the act of the physician faced with the diversity of etiologies that determine the state of shock and increase morbidity and mortality, especially in post cardiac surgery patients, in whom there are no management emergency postsurgical algorithms. In view of this situation, at the National Institute of Cardiology Ignacio Chávez, a management algorithm has been made and improved in cardiac postsurgical patients: through focused ultrasonography, including transthoracic echography, pulmonary ultrasound, optic nerve ultrasound, and renal ultrasound by evaluating renal resistive indices. Several societies have created their protocols for addressing patients in critical condition,
\end{abstract}

\section{Correspondencia:}

*Jessica Garduño-López

Belisario Domínguez, 1

Col. Sección XVI, Del. Tlalpan

Fecha de recepción: 16-11-2018

Fecha de aceptación: 10-01-2019

C.P. 14080 , Ciudad de México, México

E-mail: jeck_7_7@ hotmail.com

DOI: 10.24875/ACM.M19000026
Disponible en internet: 08-05-2019 Arch Cardiol Mex. 2019;89(2):138-149 www.archivoscardiologia.com

1405-9940 @ 2019 Instituto Nacional de Cardiología Ignacio Chávez. Publicado por Permanyer México SA de CV. Este es un artículo Open Access bajo la licencia CC BY-NC-ND (http://creativecommons.org/licenses/by-nc-nd/4.0/). 
so in the Institute, specifically in cardiovascular intensive therapy, has created the CCROSS protocol (Cardiac, Cerebral, Renal, Optic nerve, lung UltraSound Study) for the initial approach of these patients, and it is being carried out a study for its validation, reproducibility and efficacy.

Key words: Focused ultrasound. Cardiac surgery. Immediate postsurgical. Intensive care unit. México.

\section{Introducción}

El manejo postoperatorio inmediato en cirugía cardíaca debe realizarse en terapia intensiva cardiovascular, y requiere un equipo multidisciplinario con cardiólogos y/o médicos especialistas en medicina crítica con alta especialidad en terapia intensiva cardiovascular, así como con conocimientos avanzados en ecocardiografía crítica y ultrasonografía crítica (pulmonar, accesos vasculares, Doppler transcraneal, etc.). La detección temprana de complicaciones derivadas de la cirugía, la circulación extracorpórea y comorbilidades asociadas influyen de manera directa en el pronóstico del paciente. El uso de la ecocardiografía, transtorácica y transesofágica, ayuda al diagnóstico diferencial en los pacientes con inestabilidad hemodinámica, es un método a la cama del paciente, seguro, de bajo costo, y que debe estar disponible en todas las unidades de cuidados intensivos cardiovasculares ${ }^{1}$.

El acceso inmediato a la ecocardiografía las 24 horas del día, los 7 días de la semana, es clave para manejar la labilidad hemodinámica que es común después de la cirugía cardíaca. La ecocardiografía transesofágica es esencial para diagnosticar la causa de la inestabilidad hemodinámica en pacientes ventilados después de la cirugía cardíaca. Un estudio ecocardiográfico puede respaldar un diagnóstico de falla del ventrículo izquierdo (VI), hipovolemia e isquemia miocárdica, o identificar complicaciones quirúrgicas cardíacas como taponamiento, obstrucción dinámica del tracto de salida del VI secundaria a movimiento sistólico de la valva de la válvula mitral anterior o fallo agudo del ventrículo derecho (VD). La ecografía pulmonar realizada al mismo tiempo que la ecografía transtorácica (ETT) agrega información diagnóstica significativa que incluye la identificación de edema pulmonar, derrame pleural, consolidación y neumotórax².

Por lo antes mencionado, los médicos intensivistas cardiovasculares deben tener formación y adiestramiento básico-avanzado en ecocardiografía crítica (transtorácica y transesofágica) ${ }^{2,3}$.

\section{Generalidades}

La recuperación de la homeostasis es uno de los objetivos primarios en el cuidado intensivo postoperatorio de la cirugía cardíaca, misma que se ve afectada por los cambios generados por el uso de la circulación extracorpórea, así como el fenómeno de isquemia-reperfusión en el corazón, hipotermia, trastornos en el sistema de coagulación, y efectos adversos de la terapia transfusional por sangrado 4 .

La valoración clínica debe complementarse con ultrasonografía enfocada, siguiendo protocolos establecidos para determinar la causa primaria del estado de choque y resolución de esta, así como posibles complicaciones a otros órganos.

En el Instituto Nacional de Cardiología Ignacio Chávez de la Ciudad de México se propone un protocolo ultrasonográfico enfocado a pacientes posquirúrgicos cardiovasculares, que tiene como objetivo detectar etiología del estado de choque y posibles complicaciones en el posquirúrgico inmediato, así como su rápida resolución. El algoritmo desarrollado se basa en la adaptación de un protocolo específico para pacientes operados de cirugía cardíaca, enfocado a un análisis secuencial para la detección de choque (cardiogénico, hipovolémico, obstructivo), valvulopatías, alteraciones de la movilidad, disfunción protésica y valoración hemodinámica no invasiva. Por otro lado, determina afecciones en otros sistemas, tales como eventos vasculares cerebrales de tipo hemorrágico 0 isquémico secundario a procedimiento quirúrgico, bomba de circulación extracorpórea, anticoagulación y predecir lesión renal aguda, mediante el índice resistivo renal.

Es importante determinar el tipo de choque y sus causas ${ }^{5}$ :

1. Choque cardiogénico. Hipoperfusión tisular, secundario a colapso circulatorio por falla de bomba cardíaca, infarto de miocardio, miocarditis fulminante, síndrome poscardiotomía, etc.

2. Choque hipovolémico. Con efecto de precarga cardíaca inadecuada secundario a pérdida del intravascular, por ejemplo; hemorragia postoperatoria, hemorragia gastrointestinal, uso excesivo de diuréticos, etc., poco aporte hídrico.

3. Choque distributivo. Coexiste una precarga cardíaca inadecuada debido a vasodilatación y fuga vascular; ejemplos típicos son el síndrome vasopléjico posbomba, el síndrome de respuesta inflamatoria sistémica posoperatoria, sepsis y la reacción anafiláctica. 
4. Choque obstructivo. Precarga cardíaca inadecuada debido a obstrucción del retorno venoso; por ejemplo, taponamiento pericárdico, neumotórax a tensión, hipertensión intraabdominal u obstrucción del flujo sanguíneo arterial (p. ej., embolia pulmonar).

\section{Alteraciones cardiohemodinámicas}

El injerto de derivación aortocoronaria (ACBG) por estenosis de la arteria coronaria izquierda, enfermedad triple vaso y angina refractaria al tratamiento médico, es el procedimiento quirúrgico más común. Otros procedimientos quirúrgicos, junto con el ACBG o solo, incluyen reparación o reemplazo de válvulas, reparación de defectos congénitos o adquiridos y reparación o reemplazo de la raíz aórtica. Menos comunes son la extirpación de tumores intracardíacos y la aneurismectomía del $\mathrm{V}^{6}$.

Mundialmente se reporta una mortalidad global de la revascularización miocárdica del $3.2 \%$. Esta parece ser influenciada por la edad, siendo en los menores de 70 años del $2.6 \%$ y en los mayores de 70 años del $6 \%$. La mortalidad en la cirugía valvular es aún mayor, siendo del 5 al 9\%. Sin embargo, la mortalidad de los pacientes con cambio valvular mitral por isquemia es del $30 \%{ }^{7}$.

La disfunción miocárdica es una consecuencia de isquemia o infarto perioperatorio, misma que requiere intervenciones específicas y posible revascularización. Corresponde al $5 \%$ de los pacientes estables y al 10\% de los inestables, con una mortalidad del 10\%,8.

\section{Sangrado mediastinal}

Es una complicación que ocurre en el 5 al $25 \%$ de los pacientes, de los cuales se reintervienen entre el 2 y el $4 \% 9,10$. Dentro de las causas de bajo gasto se debe descartar el sangrado quirúrgico activo de las líneas de sutura o arterial. Si el sangrado es mayor de $10 \mathrm{ml} /$ $\mathrm{kg}$ en la primera hora, $8 \mathrm{ml} / \mathrm{kg}$ en la segunda hora, $6 \mathrm{ml} / \mathrm{kg}$ en la tercera hora, o si suma más de $20 \mathrm{ml} / \mathrm{kg}$ en las 4 primeras horas o se produce un aumento súbito de la cantidad de sangrado, cumple los criterios de reintervención ${ }^{9,10}$. Christensen define como sangrado severo posquirúrgico el mayor a $200 \mathrm{ml} / \mathrm{h}$ a $1,500 \mathrm{ml}$ en las primeras $8 \mathrm{~h}^{11}$.

Otra definición alternativa considera el sangrado $>400 \mathrm{ml}$ en la primera hora, $300 \mathrm{ml}$ en la primeras dos horas y $200 \mathrm{ml}$ durante tres horas consecutivas ${ }^{12}$.

Así mismo, otro panel de expertos define el sangrado severo posquirúrgico como la pérdida de sangre a través de tubo endopleural mayor a 1,001-2,000 $\mathrm{ml} / \mathrm{h}$ en las primeras $12 \mathrm{~h}$ o transfusión de 5-10 unidades de paquetes globulares o plasma fresco congelado ${ }^{13}$.

\section{Taponamiento cardíaco}

Debe sospecharse en el contexto de bajo gasto cardíaco postoperatorio. Este puede resultar de una colección relativamente pequeña de líquido pericárdico posterior con compresión asociada de una cámara cardíaca adyacente o una cámara localizada. En el periodo postoperatorio temprano, las colecciones pericárdicas suelen ser de sangre o coágulo sin drenar. El diagnóstico puede ser complicado, ya que signos clásicos, como el pulsus paradoxus, suelen estar ausentes, según la literatura su incidencia es del $1.9 \%$. Se pueden encontrar colecciones posteriores en el $66 \%$ de los casos y difusas en el $34 \%$ de los pacientes. El colapso de la aurícula derecha es evidente en el $34 \%$, de VD en el $27 \%$, y el colapso ventricular izquierdo diastólico está presente hasta en el $65 \%$ de los casos. El hallazgo más frecuente es la disminución de flujo diastólico mitral y aórtico durante la inspiración ${ }^{10}$. La presión venosa central no necesita elevarse, aunque la presión venosa central en aumento frente a la hipotensión y el bajo gasto cardíaco con aumento progresivo de vasopresores e inotrópicos, debe ser sospecha de tamponade. La sospecha de acumulación de líquido pericárdico también se justifica cuando cesa bruscamente el drenaje del tubo torácico, situación a la que nos vemos enfrentados en los pacientes posquirúrgicos. La ecocardiografía de emergencia puede ser útil, aunque la sensibilidad de la ecocardiografía transtorácica es pobre, e incluso un ecocardiograma transtorácico «normal» no puede excluir el tamponade, por lo que está indicado un ecocardiograma trasesofágico $^{14,15}$. Sin embargo, la exploración física y el comportamiento hemodinámico son la principal clave de sospecha de esta patología.

\section{Falla de ventrículo izquierdo}

Puede deberse a disfunción transitoria («aturdimiento") como resultado de un tiempo prolongado de bomba de circulación extracorpórea (BCE) y tiempos de pinzamiento aórtico, malperfusión coronaria, patología valvular y cambios en la poscarga o precarga. Instrumentos como la ecocardiografía puede ayudar a evaluar el estado del volumen y también a identificar anomalías de la pared segmentaria compatibles con isquemia y estenosis de la válvula o insuficiencia. La optimización de la precarga y la poscarga es esencial. Se puede 
indicar angiografía coronaria o reintervención quirúrgica para su revascularización. Si estas medidas no logran restaurar una perfusión adecuada, se debe considerar el soporte mecánico circulatorio ${ }^{15,16}$.

\section{Falla de ventrículo derecho}

Puede ser provocada por un aturdimiento post bypass, una malperfusión coronaria o una falla del VI. EI VD es susceptible a cambios agudos en la poscarga y la precarga, y un aumento repentino en cualquiera de ellos puede precipitar una falla aguda del VD. El catéter de flotación de arteria pulmonar y ecocardiograma transtorácico son extremadamente útiles para evaluar la función de $V D^{15,17}$.

\section{Choque poscardiotomía}

Se define como un índice cardíaco $<2.2 \mathrm{l} / \mathrm{min} / \mathrm{m}^{2}$, con adecuada precarga. Puede ser secundario a fracaso ventricular izquierdo $\mathrm{y} / \mathrm{o}$ derecho y asociarse o no a congestión pulmonar. La presión arterial es normal o baja, con cuadro clínico compatible con bajo gasto cardiaco (GC): oliguria (diuresis inferiores a $0.5 \mathrm{ml} / \mathrm{kg} / \mathrm{h}$ ), saturación venosa central $<60 \%$ (con saturación arterial normal) $\mathrm{y} / \mathrm{o}$ lactato $>3 \mathrm{mmol} / \mathrm{l}$, sin hipovolemia. Tiene una incidencia del 8 al 10\% con una mortalidad de hasta el $70 \%$. Su diagnóstico diferencial es recalentamiento, anemia extrema, reacción alérgica, efecto farmacológico, sepsis hiperaguda, insuficiencia suprarrenal, hipertiroidismo o pancreatitis. Se caracteriza por disminución en las concentraciones de vasopresina endógenas. Su manejo incluye la optimización de la precarga y la utilización de vasopresores como noradrenalina y vasopresina ${ }^{15,18}$.

Está demostrado que la cirugía cardíaca incrementa el estado metabólico, lo cual produce desequilibrio entre el aporte y la demanda, especialmente en presencia de disfunción cardíaca y reserva cardiovascular limitada. El soporte cardiovascular posquirúrgico tiene como objetivo minimizar cualquier alteración sistémica o local entre el aporte y el consumo de oxígeno $\left(\mathrm{VO}_{2}\right)$. Un incremento del $\mathrm{VO}_{2}$ entre el $10 \mathrm{y}$ el $20 \%$ ocurre en las primeras horas del posquirúrgico y permanece hasta las $48 \mathrm{~h}$, especialmente en pacientes con soporte mecánico ventilatorio, dolor, ansiedad y delirio ${ }^{1,19}$.

\section{Alteraciones pulmonares}

Las complicaciones respiratorias son frecuentes en los pacientes posquirúrgicos de cirugía cardíaca y pueden ser consecuencia de factores inflamatorios secundarios a BCE, factores mecánicos y hemodinámicos (edema pulmonar cardiogénico por falla ventricular izquierda, valvulopatía mitral residual estenosis 0 insuficiencia) ${ }^{20}$.

Los cambios posquirúrgicos en la función pulmonar se relacionan con múltiples factores: anestesia general, relajación neuromuscular, esternotomía y drenajes mediastínicos y pleurales, edema pulmonar por hemodilución, sobrecarga de fluidos y disminución en la presión oncótica, edema intersticial provocado por la respuesta inflamatoria sistémica, daño pulmonar provocado por las transfusiones o por disfunción ventricular y compromiso hemodinámico ${ }^{15,20}$.

La disfunción pulmonar por la respuesta inflamatoria es bien tolerada en la mayoría de los pacientes y se manifiesta con la disminución de la $\mathrm{PaO}_{2} / \mathrm{FiO}_{2}$, de la distensibilidad alveolar y aumento del agua pulmonar total. Los pacientes que desarrollan lesión pulmonar aguda se benefician de medidas de protección alveolar y en su caso de medidas de reclutamiento alveolar, para disminuir complicaciones secundarias a lesión pulmonar asociada a ventilación mecánica ${ }^{21,22}$.

Es frecuente también la aparición de derrames pleurales que no se deben puncionar a menos que causen deterioro respiratorio, o bien derrames pleurales mayores del $50 \%{ }^{23}$.

El desarrollo de atelectasia posquirúrgica es etiológicamente multifactorial ${ }^{24,25}$ :

- Compresión del lóbulo inferior izquierdo en la disección de la arteria mamaria izquierda.

- Parálisis frénica izquierda que ocurre en el $2.1 \%$ de los pacientes que reciben hipotermia tópica con la consecuente disfunción mecánica.

- Atelectasias residuales después del colapso al suspender la ventilación mecánica durante la BCE.

- Disminución del surfactante alveolar. La predisposición a atelectasias se incrementa en el postoperatorio por la presencia de dolor y los tubos de drenaje.

La tromboembolia pulmonar ocurre en el $3.2 \%$ de los pacientes, casi exclusivamente en los de revascularización, con una mortalidad del 19\%24,25.

Se debe por lo tanto valorar la etiología de las alteraciones respiratorias, tanto clínicamente como mediante la utilización del ultrasonido pulmonar enfocado, sustentadas en el consenso internacional basado en la evidencia en ultrasonografía enfocada en pulmón, publicado por Volpicelli ${ }^{26}$. 

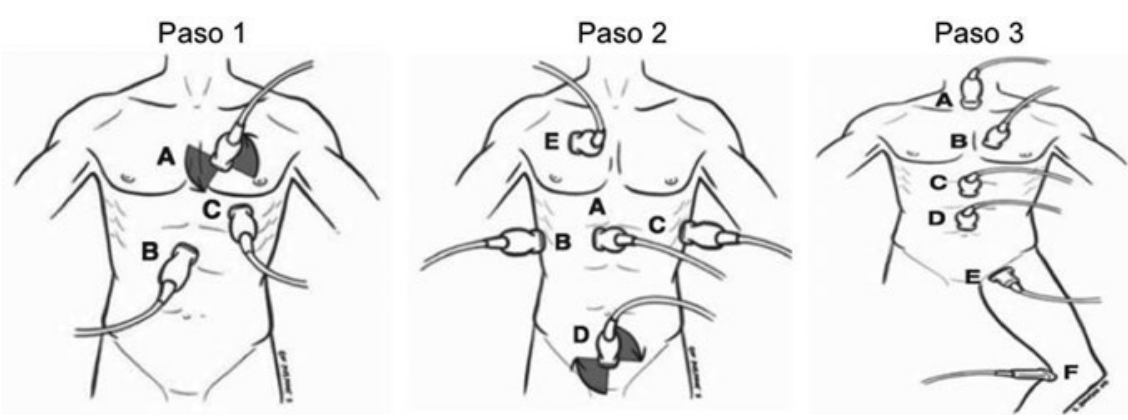

Figura 1. Ultrasonido rápido en choque (RUSH). Paso 1, evaluación de la «bomba»: vista paraesternal eje corto/largo (A), vista subxifoidea (B), vista apical (C). Paso 2, evaluación del "tanque»: eje largo IVC (A), vista pleural FAST (B), vista pleural FAST (C), pelvis/FAST (D), edema pulmonar, neumotórax (E). Paso 3, evaluación de las "tuberías»: aorta supraesternal $(A)$, aorta paraesternal $(B)$, aorta epigástrica $(C)$, aorta supraumbilical $(D)$, femoral $(E)$, poplítea $(F)$ (adaptado de Perera, et al., 201027).

\section{Protocolo CCROSS (Cardiac, Cerebral, Renal, Optic nerve, lung ultraSound Study)}

En el Instituto Nacional de Cardiología Ignacio Chávez se propone un protocolo ultrasonográfico enfocado a pacientes posquirúrgicos cardiovasculares, con el objetivo de detectar etiologías causales del estado de choque en estos pacientes. Se basa en la implementación de protocolos descritos en la literatura, utilizados en pacientes en estado de choque, abarcando: choque hipovolémico; consecuencia de sangrado por mala técnica quirúrgica o coagulopatía inducida, hemotórax, choque cardiogénico; posbomba, o por compromiso incidental de circulación coronaria, infarto perioperatorio, choque obstructivo, y neumotórax incidental por colocación de dispositivos transquirúrgicos. Por otro lado, también se persigue determinar afecciones en otros sistemas, tales como eventos vasculares cerebrales de tipo hemorrágico 0 isquémico secundario a procedimiento quirúrgico, bomba de circulación extracorpórea, anticoagulación, y al mismo tiempo y de forma temprana, predecir lesión renal aguda, mediante el índice resistivo renal.

\section{Protocolo RUSH y protocolo FALLS}

El Protocolo RUSH (Rapid Ultrasound in Shock) y el protocolo FALLS (Fluid Administration Limited by Lung Sonography) se basan en el manejo del choque de origen no determinado basado en la observación y hallazgos del ultrasonido torácico, mediante la evaluación de tres pasos ${ }^{27}$ (Fig. 1):

1. La «bomba», llamada así a la determinación de la función cardíaca, de tal forma que el primero en evaluar es el espacio pericárdico, para determinar si el paciente

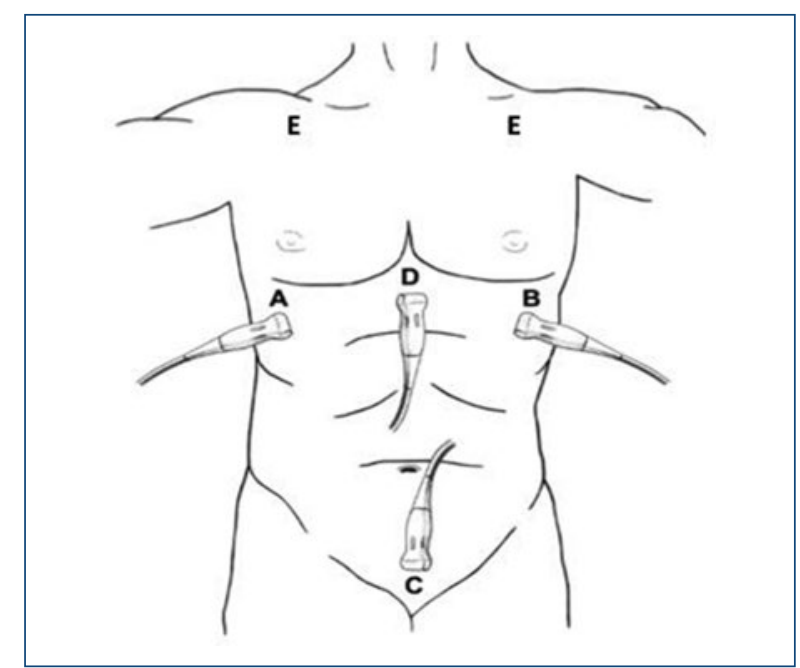

Figura 2. Las cuatro vistas del escaneo Focus Assesment with Sonography for Trauma (FAST). A: cuadrante superior derecho. B: cuadrante superior izquierdo. C: vista suprapúbica. D: vista subxifoidea del corazón (adaptado de Giraldo-Restrepo, et al., 201532).

eFAST: extendido a ápices pulmonares.

tiene derrame que comprometa el estado hemodinámico. Segundo, se evalúa el VI para analizar la contractilidad global, determinación del tamaño y estado de la contractilidad en caso de choque cardiogénico. Tercero, se centra en determinar el tamaño relativo del VI en comparación con el ventrículo derecho, lo cual es primordial para descartar compromiso de cavidades derechas e interdependencia ventricular ${ }^{27}$.

2. El «tanque», concepto conferido a determinar la condición de volumen intravascular mediante la vena cava inferior, por medio del tamaño y el índice de 


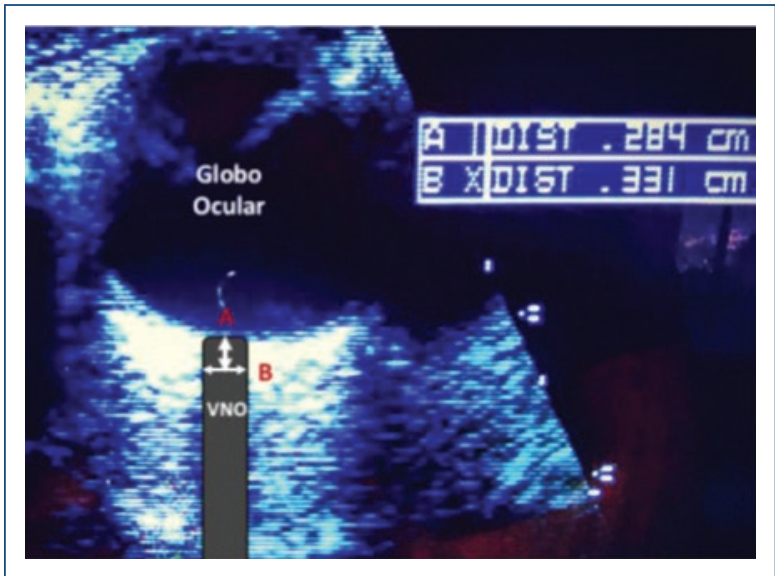

Figura 3. Vaina del nervio óptico (VNO). A: línea de referencia vertical desde la unión del nervio óptico con el globo ocular a $3 \mathrm{~mm}$. B: corte transversal perpendicular de la VNO.

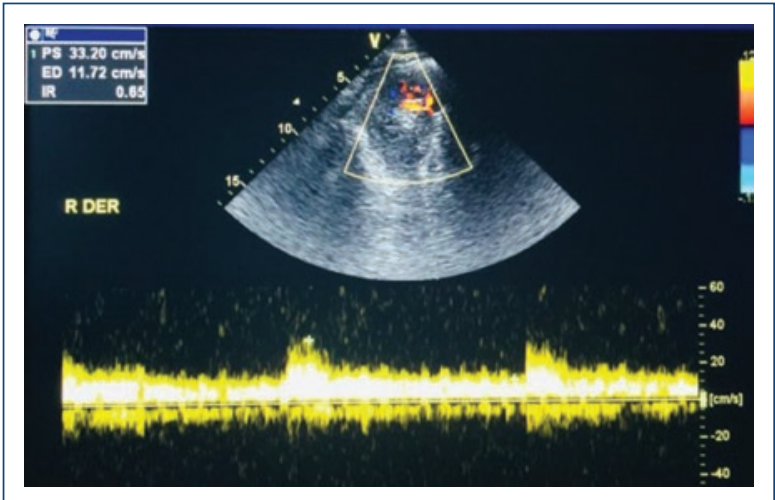

Figura 4. Curva obtenida por ultrasonografía Doppler del flujo sanguíneo. En la parte superior de la figura, patrón de flujo de arterias interlobares y arcuatas.

colapsabilidad, lo cual nos hace sospechar de choque hipovolémico y respuesta a volumen positivo ${ }^{27,28}$.

3. Las «tuberías», el último concepto que permite la evaluación de las grandes arterias y venas del cuerpo, conocido como «las tuberías rotas u obstruidas", en busca de aneurisma abdominal y/o trombosis venosa profunda, esta última cuando existe falta de compresión venosa total poplítea o femoral, misma que además sugiere en un paciente hipotenso tromboembolia pulmonar masiva ${ }^{28}$.

\section{Protocolo BLUE}

El protocolo BLUE (Bedside Lung Ultrasound in Emergency) permite el diagnóstico de insuficiencia respiratoria aguda. El edema pulmonar, embolia pulmonar,
Tabla 1. Factores de riesgo para el accidente cerebrovascular (ACV) temprano y tardío después de la cirugía cardiovascular

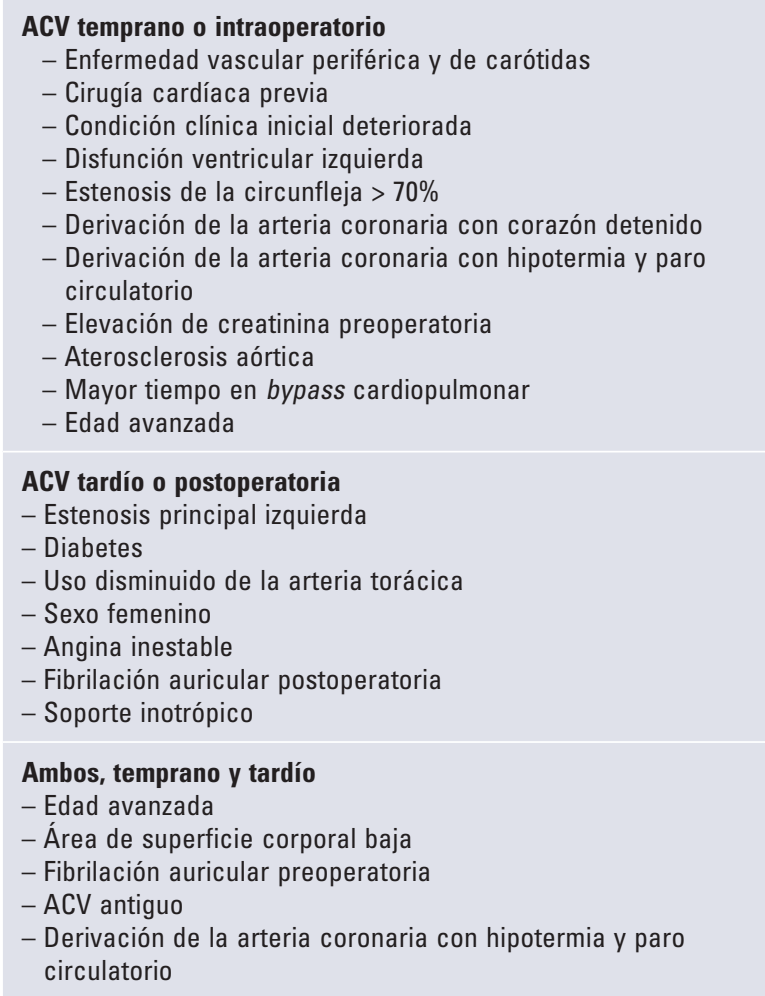

neumonía, enfermedad pulmonar obstructiva crónica, asma y el neumotórax producen perfiles específicos, que pueden ser sospechados por medio de la implementación de este protocolo ${ }^{29}$ (Figura 5).

\section{Protocolo FALLS}

EI Protocolo FALLS (Fluid Administration Limited by Lung Sonography) adapta el protocolo BLUE a la falla circulatoria aguda. Realiza la búsqueda secuencial de choque obstructivo, cardiogénico, hipovolémico y distributivo mediante ecocardiografía simple en tiempo real y luego ecografía pulmonar para evaluar un parámetro directo de la volemia clínica: la aparición de líneas $B$, que sugieren la presencia de síndrome intersticial, se considera como el punto final para la terapia de fluidos ${ }^{30,31}$ (Figura 6).

\section{FAST y eFAST}

FAST y eFAST (extended Focused Assessment with Sonography for Trauma) puede ayudar a identificar líquido libre sugestivo de hemoperitoneo, hemotórax, hemopericardio, neumotórax, hemotórax 


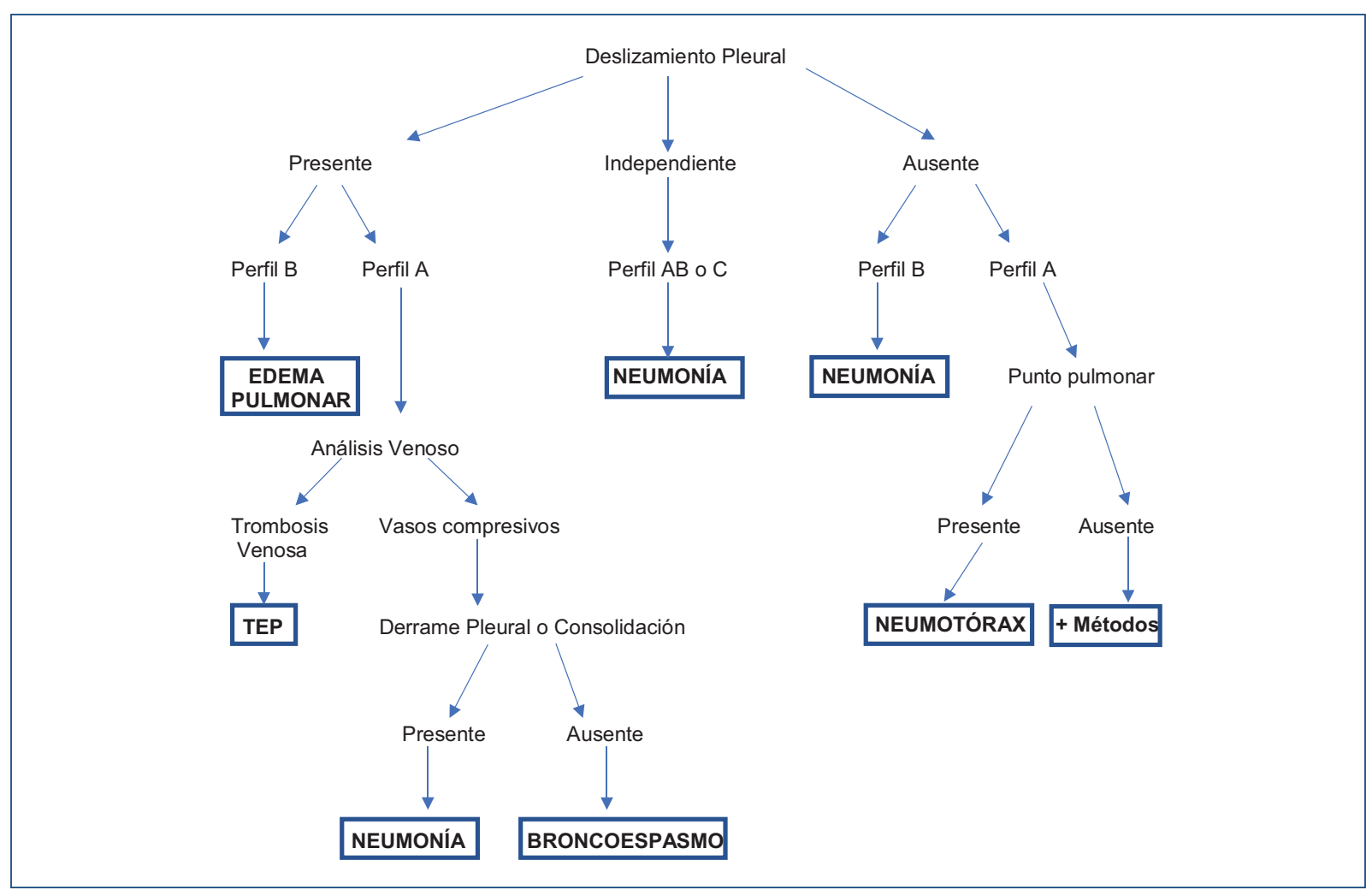

Figura 5. Protocolo BLUE. Algoritmo para valorar la insuficiencia respiratoria aguda de acuerdo con los patrones ultrasonográficos pulmonares. Perfil A: presenta deslizamiento pleural y líneas A. Perfil AB: perfil B, deslizamiento pleural en asociación con líneas B. Perfil B: presencia de líneas $B$, semideslizamiento pleural. Perfil C: presencia de consolidaciones o áreas de atelectasia, asociada o no a derrame pleural (adaptada de Lichtenstein, $2014^{29}$ ).

y atelectasia ${ }^{32-35}$. La sensibilidad varía entre el 69 y el $98 \%$ para la detección de líquido libre, y del $63 \%$ para la detección de lesión de órgano sólido ${ }^{32}$, en cuanto a la especificidad es alta para la detección de líquido libre y órganos sólidos (94-100\%). La sensibilidad de eFAST para el neumotórax y el hemotórax es más alta que la de la radiografía de tórax (11-21 frente a 43-77\%) ${ }^{32,33}$. Se evalúa la ventana hepatorrenal, esplenorrenal, pélvica, subxifoidea y ápices pulmonares $^{32-34}$ (Fig. 2).

\section{Protocolo CCROSS, complicaciones neurológicas y medición ultrasonográfica de la vaina del nervio óptico}

Las complicaciones neurológicas han sido una preocupación importante a lo largo de la historia de la cirugía cardíaca ${ }^{36}$.

Se producen complicaciones neurológicas, que incluyen ictus isquémico, lesión cerebral hipóxica isquémica, delirio y deterioro cognitivo, que conllevan una alta morbilidad y mortalidad. Por otra parte la hipertensión intracraneal, como complicación frecuente en los enfermos con lesión cerebral aguda, requiere de un diagnóstico y tratamiento temprano y oportuno para asegurar una mejor evolución $n^{37,38}$.

La incidencia de ictus isquémico postoperatorio (predominantemente tromboembólico) es de hasta el $4 \%$ para la revascularización coronaria y puede aumentar al $10 \%$ para los reemplazos valvulares y los procedimientos combinados, y este puede aumentar aún más con la presencia de fibrilación auricular perioperatoria ${ }^{39}$.

El delirio, a menudo del tipo hipoactivo, se desarrolla en el 25 al $50 \%$ de los pacientes después de una cirugía cardíaca y se asocia con deterioro cognitivo y aumento de la mortalidad 40 .

Existen factores de riesgo para el accidente cerebrovascular temprano y tardío después de la cirugía cardiovascular ${ }^{41,42}$ (Tabla 1):

En la actualidad, disponemos de otras formas de valoración neurológica, más tempranas y menos invasivas, como lo es la medición del nervio óptico. 


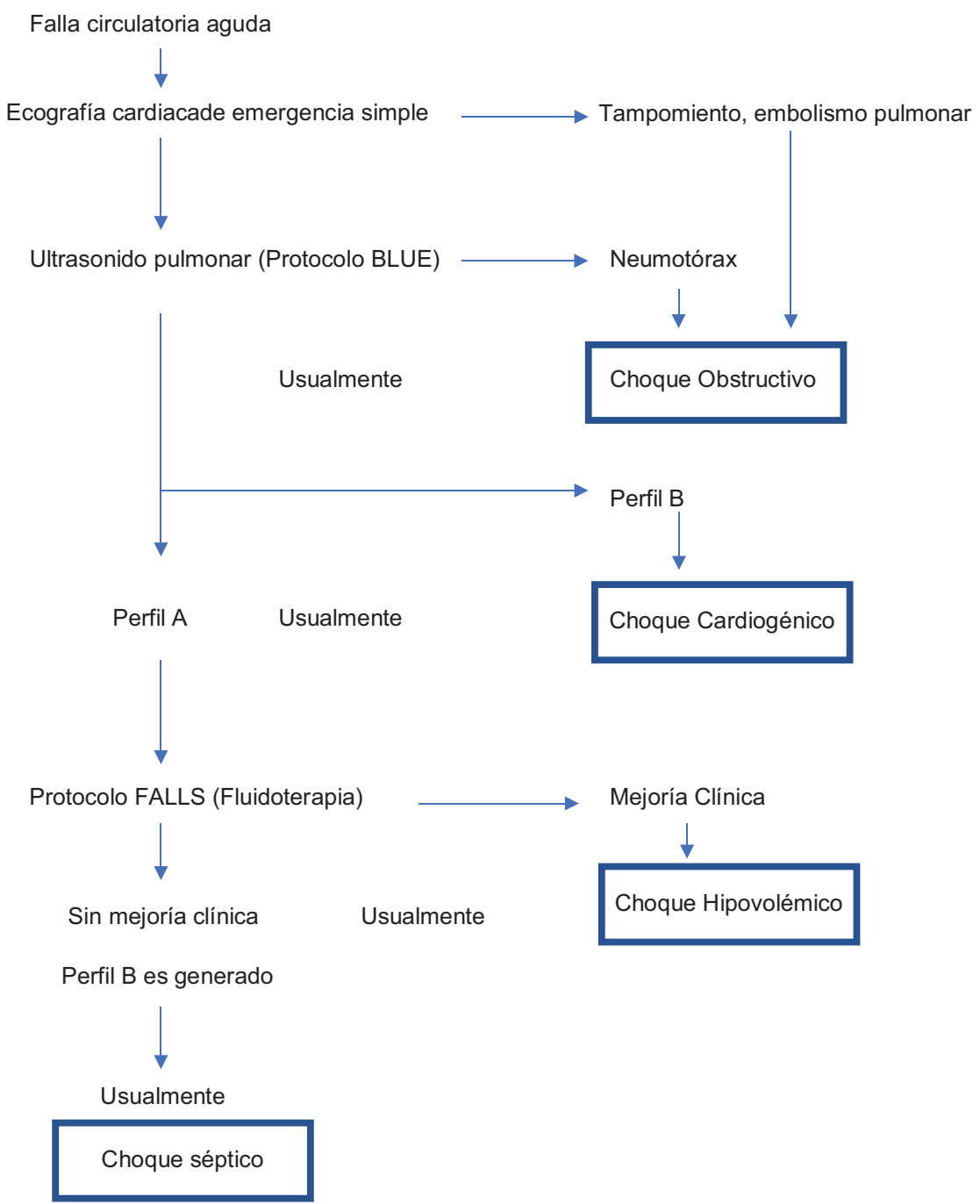

Figura 6. Administración de líquidos limitada por ecografía pulmonar: el lugar de la ecografía pulmonar en la evaluación de la insuficiencia circulatoria aguda (protocolo FALLS) (adaptada de Lichtenstein, 201230).

Este es una prolongación del sistema nervioso central y, por lo tanto, está recubierto por meninges y líquido cefalorraquídeo, concepto anatómico que explica el papiledema que se presenta con el incremento en la presión intracraneana (PIC) y fundamento de los cambios en el diámetro de la vaina del nervio óptico como reflejo de las fluctuaciones de la $\mathrm{PIC}^{38}$.

En 1965 se realizó el primer reporte de ultrasonografía del ojo. Hansen y Helmke postularon en 1997 por qué el incremento del diámetro de la vaina del nervio óptico (DVN) tiene una estrecha correlación con el incremento de la $\mathrm{PIC}^{43}$.

Recientemente se ha propuesto que la medición del diámetro de la vaina del nervio óptico a través de la ventana ocular puede ser un método no invasivo para la detección de hipertensión endocraneana, mediante la utilización de un transductor lineal de alta frecuencia (7-10 mhz). La explicación de esta medición se encuentra en que la porción más distal del nervio óptico está recubierta por la duramadre, formando una membrana conocida como vaina del nervio óptico ${ }^{44}$.

Ultrasonográficamente, el nervio óptico se identifica como la estructura hipoecoica de trayecto regular posterior al globo ocular. El estándar de medición requiere trazar una línea vertical que se inicia en la unión del nervio óptico con el globo ocular; esta línea es simplemente una referencia y debe medir $3 \mathrm{~mm}$. 


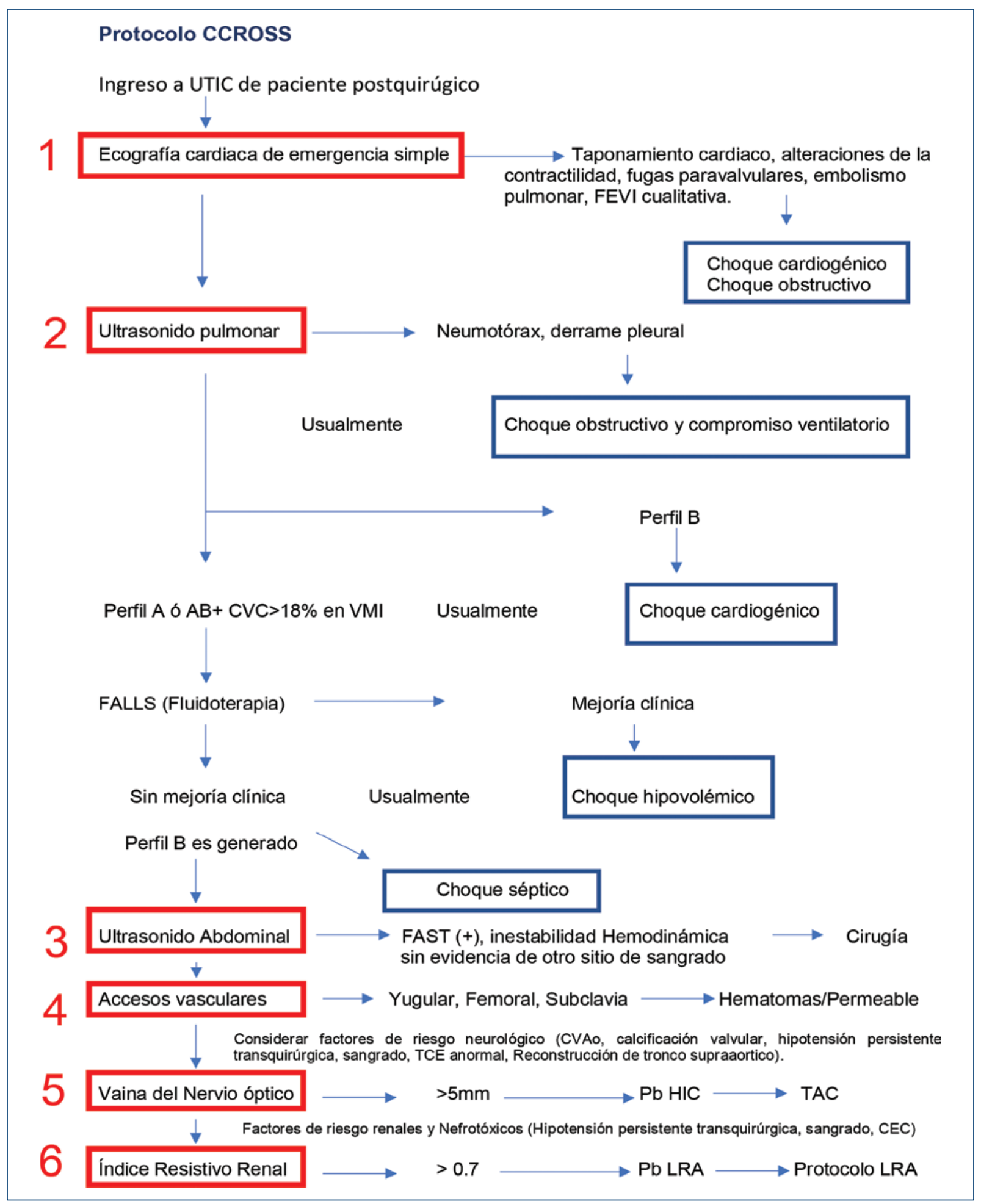

Figura 7. Protocolo CCROSS (Cardiac, Cerebral, Renal, Optic nerve, lung ultraSound Study).

Posteriormente se traza una línea horizontal de borde a borde del nervio óptico, esta segunda línea es la que mide el valor en milímetros del nervio óptico ${ }^{43,44}$. Para la mayoría de los autores revisados $5 \mathrm{~mm}$ es el punto de corte para que el estudio se considere positivo para hipertensión endocraneana ${ }^{45}$ (Fig. 3). 
Hoja de datos sugerida para la valoración ultrasonográfica enfocada

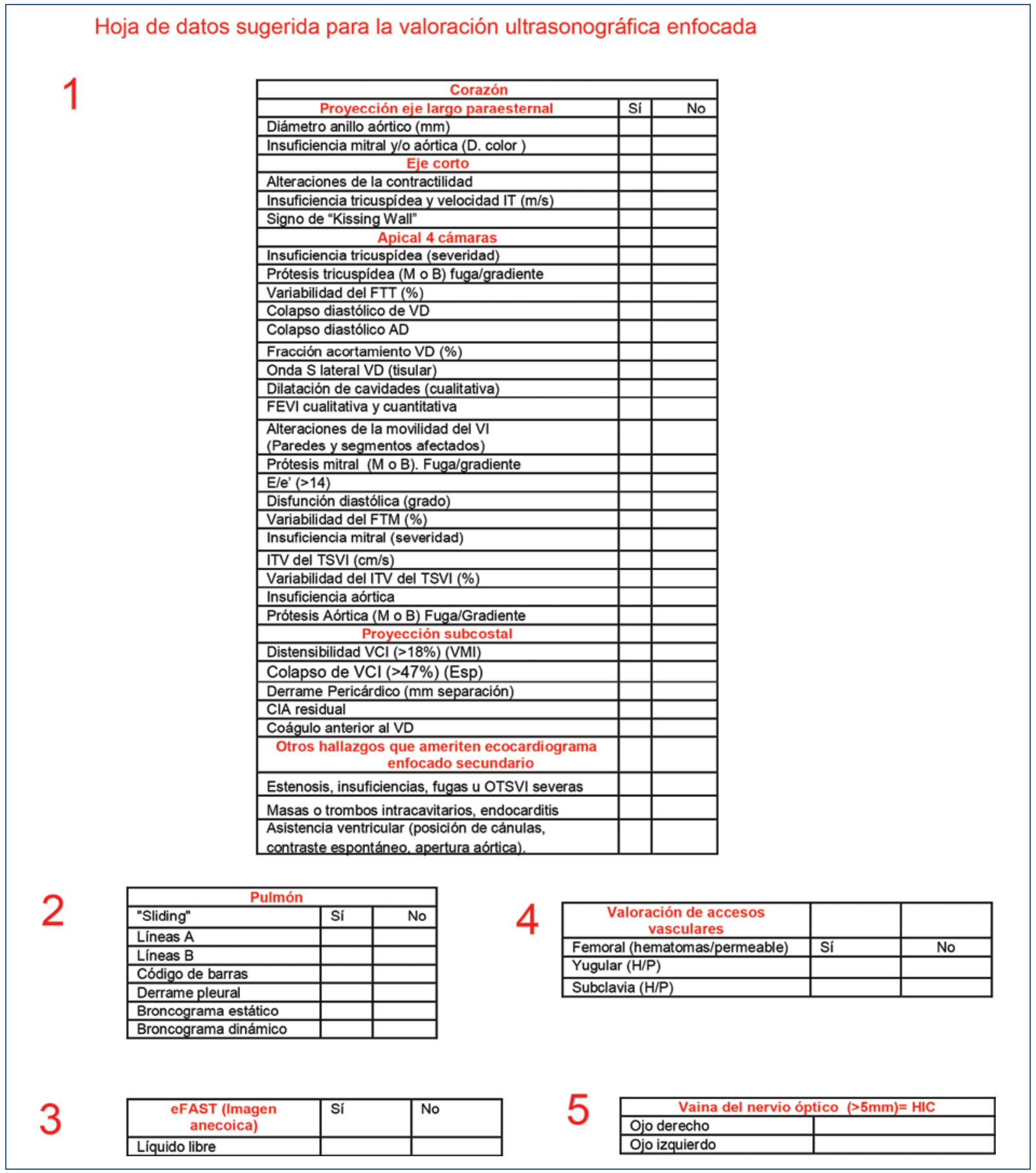

Figura 8. Hoja de datos sugerida para la valoración ultrasonográfica enfocada.

IT: insuficiencia tricuspídea; M: mecánica: B: biológica; FTT: flujo transtricuspídeo; VD: ventrículo derecho; VI: ventrículo izquierdo; $A D$ : aurícula derecha; FEVI: fracción de eyección del ventrículo izquierdo; ITV de TSVI: variabilidad del tracto de salida del ventrículo izquierdo; VCl: vena cava inferior; CIA: comunicación interauricular; OTSVI: obstrucción del tracto de salida del ventrículo izquierdo; H/P: hematoma, permeable.

\section{Protocolo CCROSS y complicaciones renales e índice resistivo renal}

La extracción hepatoesplénica y renal de oxígeno se incrementan hasta en un $50 \%$ en el posquirúrgico inmediato de cirugía cardíaca. La lesión renal aguda es una de las complicaciones más frecuentes en el enfermo grave, ocurre en un $30 \%$ de los pacientes posquirúrgicos cardíacos, que se asocia a una elevada morbimortalidad $^{46}$. 
Los factores de riesgo asociados con la aparición de lesión renal aguda después de cirugía cardíaca incluyen: edad, hipertensión arterial previa, lesión renal preexistente, transfusión sanguínea, soporte inotrópico, duración de BCE, pinzamiento aórtico, hipoxia y hemodilución severa ${ }^{47}$.

La evaluación de la lesión renal aguda se realiza de manera convencional por medio de variables estáticas, las cuales se basan en marcadores tardíos de lesión renal como el volumen urinario, el filtrado glomerular y, aún más tardío, el nivel de creatinina. El índice resistivo renal (IRR) es un marcador de evaluación ultrasonográfica temprana de lesión renal aguda que se caracteriza por ser accesible, reproducible, cuantificable, dinámico y con una elevada sensibilidad, especificidad y capacidad pronóstica ${ }^{48}$.

Anatómicamente, las arterias renales derecha e izquierda son ramas directas de la aorta abdominal y se dividen a nivel del hilio renal en posterior y anterior; estas se dividen en arterias segmentarias y a su vez en arterias interlobares localizadas periféricamente rodeando las pirámides renales que al llegar a la unión corticomedular se dividen en arterias arcuatas. Son precisamente estas últimas ramas arteriales donde se mide la velocidad de flujo arterial. Se utiliza una técnica ultrasonográfica modo 2D para localizar el riñón y la técnica Doppler color para visualizar la irrigación renal. Se localizan las arterias de la unión corticomedular renal, y utilizando Doppler pulsado se genera una curva, la cual tiene un pico sistólico seguido de una meseta y finalmente el pico diastólico, el cual genera una curva característica ${ }^{49}$ (Fig. 4).

El IRR medido por ultrasonografía Doppler fue descrito por Pourcelot con la siguiente fórmula:

IRR= Velocidad sistólica máxima - Velocidad diastólica final

Velocidad sistólica máxima

El IRR es una herramienta útil para evaluar la resistencia vascular renal y se considera normal un rango comprendido de 0.60 a 0.70 . Se recomienda la medición seriada (tres ocasiones) y hacer un promedio, no debe existir variabilidad $>10 \%$ para que se considere normal y reproducible ${ }^{50}$.

Existen otras variables que pueden generar confusión y modificar el valor del IRR. Destacan la disminución de la distensibilidad arterial, el incremento de la presión arterial, la frecuencia cardíaca, la edad, la presión intraabdominal y la existencia de lesión renal aguda (LRA), por lo que se debe poner especial atención en estas, ya que son variables que se alteran durante el posquirúrgico, incluida la cirugía cardíaca ${ }^{51}$.

\section{Conclusiones}

Esta revisión se realizó con el objetivo de plantear la necesidad de la creación de un nuevo protocolo ultrasonográfico enfocado en la valoración inicial de los pacientes posoperados de cirugía cardíaca, los cuales representan un reto para el clínico que se desenvuelve en la terapia intensiva cardiovascular. Actualmente, el ultrasonido es una herramienta de gran ayuda para el abordaje y toma de decisiones en los pacientes en estado crítico. Diversas sociedades han creado sus protocolos de abordaje, por lo que en el Instituto $\mathrm{Na}$ cional de Cardiología Ignacio Chávez, específicamente en la terapia intensiva cardiovascular, ha creado el protocolo CCROSS (Cardiac, Cerebral, Renal, Optic nerve, lung ultraSound Study) para el abordaje inicial de estos pacientes y se encuentra llevando a cabo actualmente un estudio para su validación, reproducibilidad y eficacia (Figuras 7 y 8 ).

\section{Financiamiento}

La presente investigación no ha recibido ayudas específicas provenientes de agencias del sector público, sector comercial o entidades sin ánimo de lucro.

\section{Conflicto de intereses}

Los autores declaran no tener conflicto de intereses.

\section{Responsabilidades éticas}

Protección de personas y animales. Los autores declaran que para esta investigación no se han realizado experimentos en seres humanos ni en animales.

Confidencialidad de los datos. Los autores declaran que han seguido los protocolos de su centro de trabajo sobre la publicación de datos de pacientes.

Derecho a la privacidad y consentimiento informado. Los autores declaran que en este artículo no aparecen datos de pacientes.

\section{Bibliografía}

1. Aneman A, Brechot N, Brodie D, Colreavy F, Fraser J, Gomersall C, et al. Advances in critical care management of patients undergoing cardiac surgery. Intensive Care Med. 2018;44(6):799-810.

2. Canty DJ, Heiberg J, Tan JA, Yang Y, Royse AG, Royse CF, et al. Assessment of image quality of repeated limited transthoracic echocardiography after cardiac surgery. J Cardiothorac Vasc Anesth. 2017;31:96572.

3. Rincón Salas JJ. Manejo de las complicaciones postoperatorias de la cirugía cardiaca en cuidados intensivos. Rev Asoc Mex Med Crit y Ter Int. 2013;27(3):172-78 
4. Scott Stephens RS, Whitman GJ. Postoperative critical care of the adult cardiac surgical patient. Part I: Routine postoperative care. Critical Care Medicine. 2015;43(7):1477-97.

5. Kumar A, Parrillo JE. Shock: Classification, pathophysiology, and approach to management. En: Parrillo JE, Dellinger R, editores. Critical care medicine: principles of diagnosis and management in the adult. $3^{\text {rd }}$ ed. Philadelphia: Mosby Elsevier; 2008. pp. 379-422.

6. Melanson P. Managment of post-op cardiac surgery patients [Internet]. Montreal, Quebec: McGill University, Critical Care Medicine [mayo de 2001]. Disponible en: https://www.mcgill.ca/criticalcare/teaching/protocols/cardiac.

7. Kahn SS, Denton TA, Czer L. Parrillo J, Dellinger R. Management of the patient after cardiac surgery. Critical Care Medicine: Principles of Diagnostic and management in Adults. $2^{\text {nd }}$ ed. ST Louis: Mosby; 2001. pp. 1-1670.

8. Barado Hualde J. Cuidados postoperatorios en cirugía cardíaca. Med Intensiva. 2002;26:192-3.

9. Vivacqua A, Koch CG, Yousuf AM, Nowicki ER, Houghtaling PL, Blackstone $\mathrm{EH}$, et al. Morbidity of bleeding after cardiac surgery: Is it blood transfusion, reoperation for bleeding, or both? Ann Thorac Surg. 2011; 91:1780-90.

10. Navarro OD. Derrame pericárdico y taponamiento cardiaco. Rev Colomb Cardiol. 2017;24(6):622.

11. Christensen MC, Krapf S, Kempel A, von Heymann C. Costs of excessive post operative hemorrhage in cardiac surgery. J Thorac Cardiovasc Surg. 2009;138:687-93.

12. Fergusson DA, Hébert PC, Mazer CD, Fremes S, MacAdams C, Murkin JM, et al.; BART Investigators. A comparison of aprotinin and lysine analogues in high-risk cardiac surgery. $\mathrm{N}$ Engl $\mathrm{J}$ Med. 2008;358:2319-31.

13. Dyke C, Aronson S, Dietrich W, Hofmann A, Karkouti K, Levi M, et al. Universal definition of perioperative bleeding in adult cardiac surgery. J Thorac Cardiovasc Surg. 2014;147:1458-63.

14. Price S, Prout J, Jaggar SI, Gibson DG, Pepper JR. 'Tamponade' following cardiac surgery: Terminology and echocardiography may both mislead. Eur J Cardiothorac Surg. 2004;26:1156-60.

15. Scott Stephens R. Postoperative critical care of the adult cardiac surgical patient. Part II: Procedure-specific considerations, management of complications, and quality improvement. Crit Care Med. 2015;43:1995-2014.

16. Mebazaa A, Pitsis AA, Rudiger A, et al. Clinical review: Practical recommendations on the management of perioperative heart failure in cardiac surgery. Crit Care 2010;14:201.

17. Itagaki S, Hosseinian L, Varghese R. Right ventricular failure after cardiac surgery: Management strategies. Semin Thorac Cardiovasc Surg. 2012; 24:188-94.

18. Hauffe T. Shock management for cardio-surgical ICU patients - The golden hours. Cardiac Failure Review. 2015;1(2):75-82.

19. Li P, Qu LP, Qi D, Shen B, Wang YM, Xu JR, et al. Significance of perioperative goal directed hemodynamic approach in preventing postoperative complications in patients after cardiac surgery: a meta analysis and systematic review. Ann Med. 2017;49:343-51.

20. Stephens RS, Shah AS, Whitman GJ. Lung injury and acute respiratory distress syndrome after cardiac surgery. Ann Thorac Surg. 2013;95: 1122-29.

21. Ng CS, Wan S, Yim AP, Arifi AA. Pulmonary dysfunction after cardiac surgery. Chest. 2002;121(4):1269-77.

22. Shapira N, Zabatino SM, Ahmed S, Murphy DM, Sullivan D, Lemole GM. Determinants of pulmonary function in patients undergoing coronary bypass operations. Ann Thorac Surg. 1990;50(2):268-73.

23. Johnson D, Hurst T, Thomson D, Mycyk T, Burbridge B, To T, et al. Respiratory function after cardiac surgery. J Cardiothorac Vasc Anesth. 1996;10:571-77.

24. Rousou JA, Parker T, Engelman RM, Breyer RH. Phrenic nerve paresis associated with the use of iced slush and the cooling jacket for topical hypothermia. J Thorac Cardiovasc Surg. 1985;89:921-25

25. Verheij J, van Lingen A, Raijmakers PG, Spijkstra JJ, Girbes AR, Jansen EK, et al. Pulmonary abnormalities after cardiac surgery are better explained by atelectasis than by increased permeability oedema. Acta Anaesthesiol Scand. 2005;49:1302-10.

26. Volpicelli G. International evidence-based recommendations for point-ofcare lung ultrasound. Intensive Care Med. 2012;38:577-91.

27. Perera P, Mailhot T, Riley D, Mandavia D. The RUSH exam: Rapid Ultrasound in Shock in the evaluation of the critically III. Emerg Med Clin N Am. 2010;28:29-56.

28. Perera P, Mailhot T, Riley D, Mandavia D. The RUSH exam 2012: Rapid Ultrasound in Shock in the evaluation of the critically ill patient. Ultrasound Clin. 2012;7:255-78.

29. Lichtenstein DA. Lung ultrasound in the critically ill. Ann Intensive Care. 2014;4:1

30. Lichtenstein D, Karakitsos D. Integrating lung ultrasound in the hemodynamic evaluation of acute circulatory failure (the fluid administration limited by lung sonography protocol). J Crit Care. 2012;27:533.

31. Weil MH, Shubin H. Proposed reclassification of shock states with special reference to distributive defects. Adv Exp Med Biol. 1971;23:13-23.

32. Giraldo-Restrepo JA, Serna-Jiménez TJ. The FAST and extended FAST examinations. Col J Anesthesiol. 2015;43(4):299-306.

33. Richards JR, McGahan JP. Focused assessment with sonography in Trauma (FasT) in 2017: What radiologists can learn. Radiology. 2017; 283(1):30-48

34. McGahan JP, Richards J, Gillen M. The focused abdominal sonography for trauma scan. J Ultrasound Med. 2002;21(7):789-800.

35. Galvan DA, Matsushima GK, Frankel HL. Ultrasound in the Surgical Intensive Care Unit. IMAJ. 2011;13:567-70.

36. Gilman S. Cerebral disorders after open-heart operations. N Engl J Med. 1965;272:489-98.

37. McDonagh DL. Neurological complications of cardiac surgery. Lancet Neurol. 2014;13:490-502.

38. Ropper A. Hiperosmolar therapy for raised intracranial pressure. N Engl J Med. 2012:367:746-52.

39. Nearman H, Klick JC, Eisenberg P, Pesa N. Perioperative complications of cardiac surgery and postoperative care. Crit Care Clin. 2014. 30:527-55.

40. Brown $\mathrm{CH}$. Delirium in the cardiac surgical ICU. Curr Opin Anaesthesiol. 2014:27:117-22

41. Tarakji KG, Sabik JF $3^{\text {rd }}$, Bhudia SK, Batizy LH, Blackstone EH. Temporal onset, risk factors, and outcomes associated with stroke after coronary artery bypass grafting. JAMA. 2011;305:381-90.

42. Hedberg M, Boivie P, Engstrom KG. Early and delayed stroke after coronary surgery - an analysis of risk factors and the impact on short- and long-term survival. Eur J Cardiothorac Surg. 2011;40:379-87.

43. Hansen $\mathrm{HC}$, Helmke K. Validation of the optic nerve sheath response to changing cerebrospinal fluid pressure: Ultrasound findings during intrathecal infusion tests. J Neurosurg. 1997;87:34-40.

44. Dubost $C$, Geeraerts T. Possible pitfalls when measuring the optic nerve sheath with sonography. J Surg Res. 2012;2(173):e43-5.

45. Ochoa Pérez L. Aplicaciones de la ultrasonografía en el sistema nervioso central para neuroanestesia y cuidado neurocrítico. Rev Colomb Anestesiol. 2015.

46. Hobson CE, Yavas S, Segal MS, Schold JD, Tribble CG, Layon AJ, Bihorac A. Acute kidney injury is associated with increased long区term mortality after cardiothoracic surgery. Circulation. 2009;119:2444-53.

47. Wang Y, Bellomo R. Cardiac surgery凶associated acute kidney injury: risk factors, pathophysiology and treatment. Nat Rev Nephrol. 2017;13:697-711.

48. Carrillo-Esper R, De la Torre-León T, Rosales-Gutiérrez AO, Carrillo-Córdova LD. Índice resistivo renal. Fundamentos e implementación en el enfermo grave. Rev Invest Med Sur Mex. 2014;21(2):68-72.

49. Le Dorze M, Bouglé A, Deruddre S, Duranteau J. Renal Doppler ultrasound: a new tool to assess renal perfusion in critical illness. Shock. 2012;37:360-5.

50. Keogan M, Kliewer M, Hertzberg B, DeLong D, Tupler R, Carroll B. Renal resistive indexes: variability in Doppler US measurement in a healthy population. Radiology. 1996;199:165-9.

51. Wan L, Yang N, Hiew C, Schelleman A, Johnson L, May C, Bellomo R. An assessment of the accuracy of renal blood flow estimation by Doppler ultrasound. Intensive Care Med. 2008;34:1503-10. 